\title{
Potential perturbation to Friedmann universes
}

\author{
Joan Josep Ferrando, Juan Antonio Morales, and Miquel Portilla \\ Departament de Física Tèrica, Universitat de Vatencia, 46100 Burjassot (Vatencia), Spain
}

(Received 7 December 1992)

\begin{abstract}
The energy-momentum tensor of perturbed Friedmann universes in the longitudinal gauge (depending on only one gravitational potential) is obtained in order to clarify the physical meaning of two important cases: (1) conformally static perturbations (when the potential is independent of time), and (2) nonstatic perturbations in the case where the potential allows a particular separation of time and space coordinates. The statement according to which the longitudinal gauge allows a description of high-densitycontrast regions is analyzed. In the conformally static case we suggest interpreting the energymomentum tensor as representing a set of particles in gravitational interaction, suitable for describing the post-recombination epoch. It is suggested that the second case be interpreted as a plasma in equilibrium with radiation, suitable for describing the period just before the recombination epoch.
\end{abstract}

PACS number(s): 98.80.Hw, 04.25.Nx

\section{INTRODUCTION}

Potential perturbations to Friedmann universes [see Eq. (1) below] are frequently used in cosmology. The perturbation is expressed by a Newtonian-like potential $\phi$, which is assumed to be small. The aim of this paper is to discuss the meaning of these metrics, analyzing the energy-momentum tensor obtained from Einstein's equations. In Sec. II the energy-momentum tensor corresponding to the form metric of the potential approximation is obtained without assuming $\phi$ to be small. In Sec. III we study the case where $\phi$ is independent of time, i.e., when we have a conformally static metric. In this spacetime, there exists a shear-free and vorticity-free observer $n$, and according to well-known results [1], any photon distribution which is isotropic with respect to the observer $n$ will be a solution to the Liouville equation, even if the mass distribution is not homogeneous. Therefore, if the distribution of photons was isotropic with respect to the observer $n$, at some epoch it will remain isotropic in the future. The relevance of this fact in cosmology has been considered elsewhere [2], where we have outlined the necessity of a revision of the Sachs-Wolfe effect [3]. In Sec. III A we consider high-density-contrast regions. In this case the square of the spatial gradients of $\phi$ may be of the same order as $\phi$, forcing us to keep second-order terms in the development of the energy-momentum tensor. In Sec. III B we show that the potential approximation, with $\phi$ independent of time and low density contrast, coincides with the Sachs-Wolfe solution [3], which is a pressureless solution in comoving coordinates. In Sec. IV the energy-momentum tensor corresponding to the case $\phi=\Psi\left(x^{i}\right) / a(\eta)$ is analyzed. Dautcourt [4] used this kind of metric, in order to determine the effect of the reionization on the cosmic microwave background, taking $\phi=\Psi\left(x^{i}\right) / a(\eta)$ and $k=0$, instead of the Sachs-Wolfe metric. McVittie [5] used $\phi=-G m / a(\eta) r$ and $k=0$ to describe a pointlike inhomogeneity immersed in an Einstein-de Sitter universe. More recently, MartinezGonzález, Sanz, and Silk [6] have proposed using them to compute anisotropies in the cosmic microwave background (CMB) produced by the effect of high-density regions. They based this on the fact that $\phi$ may be small even in regions of high density contrast. Here we show that the Dautcourt metric has a different meaning than the Sachs-Wolfe metric.

\section{COMPUTATION OF THE ENERGY-MOMENTUM TENSOR}

In this section we shall compute the energy-momentum tensor for the metric form

$$
d s^{2}=a^{2}\left\{-(1+2 \phi) d \eta^{2}+(1-2 \phi) h_{i j} d x^{i} d x^{j}\right\},
$$

$a=a(\eta)$ being the expansion factor of a Friedmann background, $\phi=\phi\left(\eta, x^{i}\right)$ an arbitrary potential, and $h_{i j}$ a metric of constant curvature,

$$
h_{i j}=\left(1+\frac{k}{4} r^{2}\right)^{-2} \delta_{i j}, k=0,+1,-1 .
$$

We assume that $-\frac{1}{2}<\phi<\frac{1}{2}$, but at present $\phi$ is not necessarily considered small. Synge [7] calculated the energy tensor of a similar metric, with Minkowskian space-time as the background, assuming $\phi$ independent of time.

Let us consider the timelike vector field $n=a^{-1}(1+2 \phi)^{-1 / 2} \partial_{\eta}$ normal to the slices $\eta=$ const. The induced metric and the extrinsic curvature of a slice are

$$
\begin{aligned}
& \gamma_{i j}=a^{2}(1-2 \phi) h_{i j}, \\
& K_{i j}=-\frac{1}{a \sqrt{1+2 \phi}} \partial_{\eta} \ln (a \sqrt{1-2 \phi}) \gamma_{i j} .
\end{aligned}
$$

These expressions imply that the metric (1) admits an umbilical synchronization (the vector field $n$ defines a shear-free and vorticity-free congruence). Recently, we studied [8] the energy tensor of this class of space-times. We shall write the energy-momentum tensor corresponding to the metric (1) in the form

$$
T_{\mu \nu}=\rho n_{\mu} n_{v}+q_{\mu} n_{v}+q_{\nu} n_{\mu}+p \gamma_{\mu v}+\pi_{\mu \nu},
$$

where $q_{\mu}$ is orthogonal to $n_{\mu}=(-a \sqrt{1+2 \phi}, 0,0,0)$ and $\pi_{\mu \nu}$ is the orthogonal traceless part of $T_{\mu \nu}$. Taking into account the results by Ferrando, Morales, and Portilla $[8]$, we get 


$$
\begin{aligned}
& \frac{8 \pi G a^{2}}{3}\left(\rho-\rho_{B}\right)=-\frac{\dot{a}^{2}}{a^{2}} \frac{2 \phi}{1+2 \phi}+\frac{\dot{\phi}}{1-4 \phi^{2}}\left[\frac{\dot{\phi}}{1-2 \phi}-2 \frac{\dot{a}}{a}\right]+\frac{1}{(1-2 \phi)^{2}}\left[\frac{2}{3} \Delta \phi+\frac{1}{1-2 \phi}(d \phi)^{2}\right]+\frac{2 k \phi}{1-2 \phi}, \\
& 8 \pi G a^{2}\left(p-p_{B}\right)=\frac{1}{1+2 \phi}\left\{\left[\frac{4 \ddot{a}}{a}-2 \frac{\dot{a}^{2}}{a^{2}}\right] \phi+\frac{2 \ddot{\phi}}{1-2 \phi}+\frac{\dot{\phi}}{1-4 \phi^{2}}\left[\frac{6 \phi-1}{1-2 \phi} \dot{\phi}+2 \frac{\dot{a}}{a}(3+2 \phi)\right]\right. \\
& \left.\quad-\frac{1}{3(1-2 \phi)^{2}}\left[8 \phi \Delta \phi+\frac{7+4 \phi+12 \phi^{2}}{1-4 \phi^{2}}\right](d \phi)^{2}\right\}-\frac{2 k \phi}{1-2 \phi}, \\
& 8 \pi G a q_{i}=-\frac{2}{\sqrt{1+2 \phi}}\left\{\left(\frac{\dot{a}}{a}+\frac{1+6 \phi}{(1-2 \phi)^{2}} \dot{\phi}\right) \frac{\partial_{i} \phi}{1+2 \phi}+\frac{\partial_{i} \dot{\phi}}{1-2 \phi}\right\}, \\
& \left.\left.8 \pi G \pi_{i j}=\frac{2}{1-4 \phi^{2}}\left\{2 \phi\left[\nabla_{i} \partial_{j} \phi-\frac{1}{3} \Delta \phi h_{i j}\right]+\frac{1+4 \phi+12 \phi^{2}}{1-4 \phi^{2}}\right] \partial_{i} \phi \partial_{j} \phi-\frac{1}{3}(d \phi)^{2} h_{i j}\right]\right\}
\end{aligned}
$$

where $(d \phi)^{2}=h^{i j} \partial_{i} \phi \partial_{j} \phi, \Delta=h^{i j} \nabla_{i} \nabla_{j}$ is the Laplacian operator for the metric $h_{i j}$, and the overdot means a derivative with respect to the conformal time $\eta$. The quantities $\rho_{B}$ and $p_{B}$ are the energy density and the pressure of the Friedmann background:

$$
\begin{aligned}
& \rho_{B}=\frac{3}{8 \pi G a^{2}}\left(\frac{\dot{a}^{2}}{a^{2}}+k\right), \\
& p_{B}=-\frac{1}{8 \pi G a^{2}}\left(2 \frac{\ddot{a}}{a}-\frac{\dot{a}^{2}}{a^{2}}+k\right) .
\end{aligned}
$$

\section{CONFORMALLY STATIC PERTURBATIONS}

Let us assume $\phi$ independent of time; then, (1) turns out to be a conformally static metric. In this case, Eqs. (6)-(9) can be written as

$$
\begin{aligned}
& \frac{8 \pi G a^{2}}{3}\left(\rho-\rho_{B}\right)=-\frac{\dot{a}^{2}}{a^{2}} \frac{2 \phi}{1+2 \phi}+\frac{1}{(1-2 \phi)^{2}}\left[\frac{2}{3} \Delta \phi+\frac{1}{1-2 \phi}(d \phi)^{2}\right]+\frac{2 k \phi}{1-2 \phi}, \\
& 8 \pi G a^{2}\left(p-p_{B}\right)=\frac{1}{1+2 \phi}\left\{\left(\frac{4 \ddot{a}}{a}-2 \frac{\dot{a}^{2}}{a^{2}}\right] \phi-\frac{1}{3(1-2 \phi)^{2}}\left[8 \phi \Delta \phi+\frac{7+4 \phi+12 \phi^{2}}{1-4 \phi^{2}}\right](d \phi)^{2}\right\}-\frac{2 k \phi}{1-2 \phi}, \\
& 8 \pi G a q_{i}=-\frac{2 \dot{a}}{a(1+2 \phi)^{3 / 2}} \partial_{i} \phi, \\
& 8 \pi G \pi_{i j}=\frac{2}{1-4 \phi^{2}}\left\{2 \phi\left(\nabla_{i} \partial_{j} \phi-\frac{1}{3} \Delta \phi h_{i j}\right)+\frac{1+4 \phi+12 \phi^{2}}{1-4 \phi^{2}}\left(\partial_{i} \phi \partial_{j} \phi-\frac{1}{3}(d \phi)^{2} h_{i j}\right)\right\} .
\end{aligned}
$$

One feature of this metric is the existence of an energy flux $q_{i}$ collinear with the gradient $\partial_{i} \phi$. This flux may be interpreted as the collective motion of matter with respect to $n$ insofar as there exists a new observer $n^{\prime}=\gamma(n+V e)$ measuring zero energy flux. But what does the vector field $n$ mean? When $\phi$ is independent of time, the metric (1) admits the conformal Killing vector $\partial_{\eta}$; according to well-known results (see [8] and references therein), this property allows the existence of isotropic radiation with respect to the observer $n$ collinear with the conformal Killing vector. So the flux $q_{i}$ is interpreted in a very natural way as the collective motion of matter with respect to an observer measuring isotropic radiation.

The vector field representing the motion of matter will just be the vector field $n^{\prime \mu}$ for which there is no energy flux. In order to determine it, let us consider a boost with velocity $V e^{\mu}$ :

$$
\begin{aligned}
& n^{\prime \mu}=\gamma\left(n^{\mu}+V e^{\mu}\right), \\
& e^{\prime \mu}=\gamma\left(V n^{\mu}+e^{\mu}\right) .
\end{aligned}
$$

The energy density, mean pressure, and energy flux with respect to the observer $n^{\prime \mu}$ are

$$
\begin{aligned}
& \rho^{\prime}=\gamma^{2}\left(\rho-2 q V+V^{2} \pi\right) \\
& p^{\prime}=p+\frac{1}{3} \gamma^{2} V^{2}\left(\rho+\pi-2 \frac{q}{V}\right), \\
& q_{\mu}^{\prime}=-\gamma\left\{-q_{\mu}+V t_{\mu \nu} e^{v}+\gamma^{2} V^{2}\left[\rho+\pi-q \frac{1+V^{2}}{V}\right) n_{\mu}\right. \\
& \left.\quad+\gamma^{2} V\left(\rho+\pi V^{2}-2 q V\right) e_{\mu}\right\}
\end{aligned}
$$

where we have introduced $t_{\mu \nu}=p \gamma_{\mu v}+\pi_{\mu v}, \pi=t_{\mu \nu} e^{\mu} e^{v}$, and $q=q_{\mu} e^{\mu}$. 
Equating $q_{\mu}^{\prime}$ to zero, one gets

$$
V=\frac{\rho+\pi}{2 q}\left[1-\left(1-\frac{4 q^{2}}{(\rho+\pi)^{2}}\right]^{1 / 2}\right]
$$

and substituting the above into (17) and (18) we have

$$
\begin{aligned}
& \rho^{\prime}=\rho-q V, \\
& p^{\prime}=p-\frac{1}{3} q V .
\end{aligned}
$$

Until now, we have given exact expressions for the energy-momentum tensor, but we have obtained only an implicit equation for determining the direction $e^{\mu}$ of the matter velocity. In order to get more tractable formulas, we shall consider $\phi$ to be an infinitesimal quantity. Let us introduce three quantities $\varepsilon_{0}, \varepsilon_{1}, \varepsilon_{2}$ such that $\phi$ and its two first derivatives verify $|\phi|=O\left(\varepsilon_{0}\right), \quad\left|\partial_{i} \phi\right|=O\left(\varepsilon_{1}\right)$, and $\left|\partial_{i} \partial_{j} \phi\right|=O\left(\varepsilon_{2}\right)$, where $\varepsilon_{0} \ll 1, \varepsilon_{1} \ll 1$. In some cases $\varepsilon_{2}$ will not be considered small-see below, Sec. III A. By considering Eq. (19) again, as well as (12)-(15), we get a manageable expression for the matter velocity:

$$
(\rho+p) V e_{\mu}=q_{\mu}+O\left(\varepsilon_{1}^{2}\right)+O\left(\varepsilon_{0} \varepsilon_{1}\right) .
$$

In the next subsections, we shall study two realistic situations in more detail: In Sec. III A we shall consider high-density-contrast regions (we mean $\delta \sim 1$ ) at scales smaller than the horizon; in Sec. III B we shall consider low-density-contrast regions $(\delta<1)$, but with no restriction on their sizes.

For the sake of simplicity, we shall consider a zerocurvature and zero-pressure background (Einstein-de Sitter universe). In this case the expansion factor can be written as $a(\eta)=2 \eta^{2} / H_{1}$, with $H_{1}$ as the present value of the Hubble constant.

\section{A. High-density-contrast small-scale inhomogeneities}

We shall derive here expressions for the energy tensor and the expansion of matter valid for regions with high density contrast (i.e., $\delta \sim 1$ ). In this case we have, according to Eq. (12), $\varepsilon_{2} \sim 1$. Furthermore, we shall assume that $\phi$ varies significantly at scales smaller than the horizon. In this case $\varepsilon_{1}^{2}$ may be of the same order as $\varepsilon_{0}$, and therefore we should keep second-order terms in the first derivatives. Neglecting $\varepsilon_{0}^{2}$ and $\varepsilon_{0} \varepsilon_{1}$ in (21) and (22) (but not $\varepsilon_{1}^{2}$ ) and taking into account expressions (12), (14), and (23), with $p_{B}=0$, one gets

$\rho^{\prime}=(1-2 \phi) \rho_{B}+\frac{1+4 \phi}{4 \pi G a^{2}} \Delta \phi+\left(\frac{9}{4}-\frac{1}{1+\delta}\right) \frac{(d \phi)^{2}}{6 \pi G a^{2}}$,

$p^{\prime}=-\frac{1}{3 \pi G a^{2}} \phi \Delta \phi-\left(\frac{7}{4}+\frac{1}{3(1+\delta)}\right) \frac{(d \phi)^{2}}{6 \pi G a^{2}}$,

and the matter velocity with respect to the observer measuring isotropic radiation is

$$
V_{i}=-\frac{2}{3} \frac{a^{2}}{\dot{a}} \frac{\partial_{i} \phi}{1+\delta} \text {. }
$$

We can use (24) to determine $\phi$ from $\rho^{\prime}$. At the first approximation, we have

$$
\Delta \phi=4 \pi G a^{2}\left(\rho^{\prime}-\rho_{B}\right)=4 \pi G a^{2} \delta \rho_{B} .
$$

With an Einstein-de Sitter background, as $\phi$ is independent of time, one gets the evolution of the density contrast $\delta=\eta^{2} \delta_{1}$, where $\delta_{1}$ is the density contrast at the present time $(\eta=1)$. This allows us to write the mean pressure $(25)$ in the form

$$
p^{\prime}=-\frac{4}{3} \phi \delta \rho_{B}-\left(\frac{7}{4}+\frac{1}{3(1+\delta)}\right) \frac{(d \phi)^{2}}{6 \pi G a^{2}} .
$$

Now let us study how the expansion of matter is perturbed. The four-velocity of matter is $n_{\alpha}^{\prime}=\left(a(1+\phi), V_{i}\right)$. Taking into account expression (26) and using the formula $\theta=\partial_{\alpha}\left(\sqrt{-g} n^{\prime \alpha}\right) / \sqrt{-g}$, we get

$$
\theta=3 \frac{\dot{a}}{a^{2}}\left[1-\frac{\eta^{2} \delta_{1}}{3\left(1+\eta^{2} \delta_{1}\right)}+\frac{\eta^{4} \delta^{i j} \partial_{i} \delta_{1} \partial_{j} \phi}{18\left(1+\eta^{2} \delta_{1}\right)^{2}}\right] .
$$

It is manifest that it is smaller than the background expansion. Let us illustrate this with an example assuming a power law for the density contrast $\delta_{1}=\left(r_{c} / r\right)^{\gamma}$. This may represent the external part of a cluster of galaxies. One has in this case $\phi=6 r^{2} \delta_{1} /(3-\gamma)(2-\gamma)$, $(d \phi)^{2}=36 r^{2} \delta_{1}^{2} /(3-\gamma)^{2}, \quad$ and $\partial^{i} \delta_{1} \partial_{i} \phi=-\gamma \delta_{1}^{2} /(3-\gamma)$. Before we proceed, it is appropriate to point out that $(d \phi)^{2}$ is certainly of the same order as $\phi$ when $\delta_{1}$ is of order 1 , as we stated at the beginning of the section. Taking these expressions into account, the expansion (28) turns out to be

$$
\theta=3 \frac{\dot{a}}{a^{2}}\left[1-\frac{\eta^{2} \delta_{1}}{3\left(1+\eta^{2} \delta_{1}\right)}-\frac{\eta^{4} \gamma \delta_{1}^{2}}{18(3-\gamma)\left(1+\eta^{2} \delta_{1}\right)^{2}}\right] .
$$

For $3>\gamma \geq \frac{36}{13}$ one has $\theta \leq 0$ when $r$ tends to zero.

In its turn, assuming a power law $\delta_{1}=\left(r_{c} / r\right)^{\gamma}$, Eq. (27) gives, for the pressure,

$$
\begin{aligned}
p^{\prime}=- & {\left[\frac{8}{(3-\gamma)(2-\gamma)}\right.} \\
& \left.+\left[\frac{7}{4}+\frac{1}{3(1+\delta)}\right] \frac{4}{(3-\gamma)^{2}}\right] \eta^{2} \rho_{B} r^{2} \delta_{1}^{2} .
\end{aligned}
$$

At first sight, the pressure (27) has two ill characteristics: (1) It does not correspond to any reasonable equation of state, and (2) it may even become negative, as Eq. (30) illustrates for $\gamma>3$ or $\gamma<2$. Both discard the interpretation as a gas in thermodynamic equilibrium. However, let us suggest that it could have a meaning in the framework of statistical mechanics. So we shall think of a set of particles (galaxies) with long-range interaction forces (gravitation). In this case we do not expect a perfect fluid energy-momentum tensor, and the possibility of negative pressures is quite naturally related to the gravitational interaction between the particles. Let us explain this important point in more detail. The system is described by $n$-particle distribution functions, satisfying the Bogoliubov-Born-Green-Kirkwood-Yvon (BBGKY) hierarchy of equations, and the energy-momentum tensor 
is a statistical average. It is known [9] that the mean pressure can be split into two components $p=p_{K}+p_{D}$, $p_{K}$ being the purely kinetic pressure and $p_{D}$ being the dynamic pressure due to two-body interactions. The dynamic pressure depends on the two-particle distribution function, and it is negative when the interaction is attractive and the statistical dependence is positive (we mean the difference between the two-particle distribution and the product of two one-particle distribution functions). We know from early studies [10,11] that a consequence of the gravitational interaction between galaxies is a certain degree of statistical dependence in its spatial distribution. Therefore a system of self-gravitating pointlike particles will usually have negative dynamic pressure and it could even have negative total mean pressure if the random mean-squared velocity is sufficiently small.

To interpret all the components of the energymomentum tensor in the framework of statistical mechanics is a hard task with many unsolved theoretical problems. One must prove that there exists a solution of the BBGKY hierarchy of equations to which an energymomentum tensor of the given form corresponds. This is far from the aim of this paper. We have given some steps in this direction elsewhere [12], and here we advance a result to suggest the kind of solution of the hierarchy needed. Let us consider a finite region, large enough to be considered a fair sample of the Universe. Taking the spatial average of Eq. (27) and assuming that a statistical interpretation is possible, we get $2 K+W=0$, where $K$ and $W$ are the kinetic and gravitational potential energies per unit of mass, respectively. From this result and taking into account the Layzer-Irvine equation $d(K+W) / d t+H(2 K+W)=0$, we conclude that any sufficiently large region of the Universe is virialized, in the sense that the total energy $K+W$ is stationary. Hence we arrive at the conclusion that if a suitable statistical interpretation of the energy-momentum tensor (12) - (15) could be set up, it would correspond to a virialized universe.

\section{B. Small-density-contrast large-scale inhomogeneities}

In the case where $\phi$ varies significantly at scales of the order of the horizon, we have $\varepsilon_{0} \approx \varepsilon_{1} \approx \varepsilon_{2}$; in consequence we shall keep only first-order terms in our general expressions. So neglecting second-order terms one has

$$
\begin{aligned}
& \rho^{\prime}=\left(1-2 \phi+\frac{1}{6} \eta^{2} \Delta \phi\right) \rho_{B}, \\
& p^{\prime}=\pi_{i j}^{\prime}=0, \\
& V_{i}=-\frac{2}{3} \frac{a^{2}}{\dot{a}} \partial_{i} \phi .
\end{aligned}
$$

Introducing [13] new coordinates $\left(\bar{\eta}, \bar{x}^{i}\right)$ defined by

$$
\begin{aligned}
& \bar{\eta}=\left(1+\frac{1}{3} \phi\right) \eta, \\
& \bar{x}^{i}=x^{i}+\frac{1}{6} \eta^{2} \partial_{i} \phi,
\end{aligned}
$$

one gets the Sachs-Wolfe [3] solution

$$
\begin{aligned}
d s^{2}=a^{2}(\bar{\eta})\left\{-d \bar{\eta}^{2}+\right. & {\left[\left\{1-\frac{10}{3} \phi\right] \delta_{i j}\right.} \\
& \left.\left.-\frac{1}{3} \bar{\eta}^{2} \frac{\partial^{2} \phi}{\partial \bar{x}^{i} \partial \bar{x}^{j}}\right] d \bar{x}^{i} d \bar{x}^{j}\right\} .
\end{aligned}
$$

Taking into account the relation $\rho_{B}(\bar{\eta})=\rho_{B}(\eta)(1-2 \phi)$, we can write Eq. (31) in the form

$$
\rho^{\prime}(\bar{\eta})=\left(1+\frac{1}{6} \bar{\eta}^{2} \Delta \phi\right) \rho_{B}(\bar{\eta}) .
$$

The density contrast $\delta$ in the comoving reference system is defined by $\delta=\left[\rho^{\prime}-\rho_{B}(\bar{\eta})\right] / \rho_{B}(\bar{\eta})$. Taking into account that $\phi$ is independent of time, one gets $\delta=\bar{\eta}^{2} \delta_{1}$ and Eq. (36) can be written as

$$
\Delta \phi=6 \delta_{1} \text {. }
$$

We have seen that the potential perturbation (1), with $\phi$ independent of time and assuming low density contrast, is equivalent to the Sachs-Wolfe metric. The results obtained in the previous subsection may then be considered as an extension of the Sachs-Wolfe metric to regions of high density contrast. Let us outline here that expression (33) for the collective motion of matter should be changed by expression (26) when reaching high-density regions. This is meaningful in cosmology for the study of distortions in redshift space [13].

\section{NONSTATIC PERTURBATIONS}

Let us assume now a particular time dependence for the potential $\phi=\Psi\left(x^{i}\right) / a(\eta)$, which was first considered by Dautcourt [4]. In this case we shall consider low density contrast; then, $\varepsilon_{0} \approx \varepsilon_{1} \approx \varepsilon_{2}$. As before, we shall take $k=0$. Substituting $\phi$ into (6)-(9) and neglecting second-order terms, we have

$$
\begin{aligned}
& 4 \pi G a^{2}\left(\rho-\rho_{B}\right)=\Delta \phi, \\
& p=p_{B}(1-\phi)-\rho_{B} \phi, \\
& 8 \pi G \pi_{i j}=0, \\
& q_{i}=0 .
\end{aligned}
$$

Kodama and Sasaki [14] and Mukhanov, Feldman, and Brandenberger [15] have given first-order energy tensor expressions for a general Friedmann perturbation. Equations (38)-(41) are compatible with their results. However, our expression (39) for the pressure is different from that given by Dautcourt [4].

As in this case we have no energy flux, we can take the vector $n^{\alpha}$ as representing the mean motion of matter. Then its expansion will be given directly by the trace of the extrinsic curvature: $\theta=-\operatorname{tr} K$. A simple calculation gives $\theta=3 \dot{a} / a^{2}+O\left(\varepsilon_{0}^{2}\right)$. Therefore it is a perturbation that leaves the background expansion unchanged.

Let us interpret the pressure (39) as corresponding to a plasma (electrons and protons) close to the equilibrium with radiation. So let us split the background density and pressure into radiation and plasma components: $\rho_{B}=\rho_{B r}+\rho_{B m}$ and $p_{B}=p_{B r}+p_{B m}$, with $\rho_{B r}=3 p_{B r}$. Expression (39) then becomes 


$$
p=p_{B r}(1-4 \phi)+p_{B m}(1-\phi)-\rho_{B m} \phi .
$$

Now, assuming $p_{B m} \ll \rho_{B m}$, the pressure turns out to be

$$
p=p_{B r}(1-4 \phi)+p_{B m}-\rho_{B m} \phi .
$$

The first term means that the radiation temperature should be of the form $T_{r}=T_{B r}(\eta)(1-\phi)$. The two remaining terms can be derived [16] from a Boltzmann distribution function $f(x, k)=C e^{-E / T_{m}}$, taking $T_{m}=T_{r}$, considering the nonrelativistic limit $\left(E=m+\mathbf{k}^{2} / 2 m\right)$, and assuming $m \phi / T_{m} \ll 1$.

\section{CONCLUSIONS}

We have obtained the energy-momentum tensor corresponding to the metric form known as the potential perturbation of a Friedmann universe. Two particular cases have been considered in detail, outlining their cosmological meaning.

(i) Potential of the form $\phi=\phi\left(x^{i}\right)$. When $\phi$ varies significantly at scales of the order of the horizon, the metric (1) represents, in regions of low density contrast, a pressureless perturbation of an Einstein-de Sitter universe. This solution was obtained first by Sachs-Wolfe in other coordinates (comoving and synchronous gauge).
When $\phi$ varies significantly at scales much smaller than the horizon, in regions of high density contrast, the metric (1) represents a Friedmann perturbation with non-null pressure. This one does not satisfy any reasonable equation of state and may even be negative. We have suggested interpreting it in the framework of statistical mechanics, considering a system of self-gravitating particles. This interpretation would be suitable for the present Universe if matter were mainly contained in galaxies.

(ii) Potential of the form $\phi=\Psi\left(x^{i}\right) / a(\eta)$, in the limit of low density contrast. In this case we have an inhomogeneous perfect fluid energy-momentum tensor, describing a plasma close to the equilibrium with isotropic radiation. So this metric form is suitable for representing the Universe just before the decoupling process of matter and radiation.

\section{ACKNOWLEDGMENTS}

We thank the Spanish D.G.I.C.Y.T. for their financial support of project No. PB90-0416. One of us (M.P.) would like to thank the Canadian Institute for Theoretical Astrophysics, where this paper was initiated with a grant from the Conselleria de Cultura de la Generalitat Valenciana.
[1] J. Ehlers, P. Geren, and R. K. Sachs, J. Math. Phys. 9, 1344 (1968).

[2] J. J. Ferrando, J. A. Morales, and M. Portilla, Phys. Rev. D 47, 1308 (1993).

[3] R. K. Sachs and A. M. Wolfe, Astrophys. J. 147, 73 (1967).

[4] A. G. Dautcourt, Mon. Not. R. Astron. Soc. 144, 255 (1969).

[5] G. C. McVittie, Mon. Not. R. Astron. Soc. 93, 325 (1933).

[6] E. Martínez-González, J. L. Sanz, and J. Silk, Astrophys. J. 355, L5 (1990).

[7] J. L. Synge, General Relativity (North-Holland, Amsterdam, 1960), pp. 206-208.

[8] J. J. Ferrando, J. A. Morales, and M. Portilla, Phys. Rev. 46, 578 (1992).

[9] H. J. Kreuzer, Nonequilibrium Thermodynamics and Its
Statistical Foundations (Oxford Science, Oxford, 1981).

[10] S. M. Fall and G. Severne, Mon. Not. R. Astron. Soc. 174, 241 (1976).

[11] S. M. Fall and W. C. Saslaw, Astrophys. J. 204, 631 (1976).

[12] J. J. Ferrando, J. A. Morales, and M. Portilla, "Statistical interpretation of Friedmann universes," report (unpublished).

[13] J. Moreno, Ph.D. thesis, Universitat de València, 1992.

[14] H. Kodama and M. Sasaki, Prog. Theor. Phys. Suppl. 78, 1 (1984).

[15] V. F. Mukhanov, H. A. Feldman, and R. H. Brandenberger, Phys. Rep. 215, 203 (1992).

[16] J. J. Ferrando, J. A. Morales, and M. Portilla, "A model of Universe with isotropic radiation," report (unpublished). 